\title{
Angioarchitectural studies of the utero-ovarian component in the camel (Camelus dromedarius)
}

\author{
R. Ghazi \\ Department of Animal Biology, School of Veterinary Medicine, Shiraz University, \\ P.O. Box 257, Shiraz, Iran
}

\begin{abstract}
Summary. In 6 adult camels (Camelus dromedarius) the uterus had 2 arteries on each side; one was the cranial uterine artery, which was a branch from the ovarian artery, and the other was the caudal uterine artery, which originated from the urogenital (vaginal) artery. There was no middle uterine artery, as is found in ruminants. The ovarian artery originated directly from the aorta and divided into 3 branches (ovarian proper, uterine and oviduct arteries) at a point approximately 5 $\mathrm{cm}$ before the ovary. The main drainage consisted of the utero-ovarian and caudal uterine veins. Although interconnections between arteries as well as between veins were numerous, no true veno-arterial anastomoses were found. These anatomical findings would suggest that there may be local unilateral uterine control of ovarian function in the camel.
\end{abstract}

\section{Introduction}

In the sheep the uterus has a local unilateral influence upon the ovary (Moor \& Rowson, 1966; Inskeep \& Butcher, 1966). The local unilateral passage of the luteolytic substance(s) from the uterus toward the ovary is dependent upon the vascular relationship of the utero-ovarian component. A luteolytic substance, prostaglandin (PG) F-2 $\alpha$, is produced in the endometrium, released via the uterine vein and is transferred from the utero-ovarian vein to the ovarian artery by a counter-current mechanism (Goding, 1974; Land, Baird \& Scaramuzzi, 1976).

Anatomically the ovarian artery is closely applied to the surface of the utero-ovarian vein in the ewe and the wall of both vessels although complete is thinnest where they are contiguous (Del Campo \& Ginther, 1974; Ghazi, 1977). Thus the anatomical relationship of the utero-ovarian vasculature in this species provides the basis for its possession of a local unilateral luteolytic pathway. Del Campo \& Ginther (1973) and Ginther (1974) consider that in guinea-pigs, hamsters, rats, cows, ewes and sows there is a typical local unilateral luteolytic pathway and a close vascular relationship of the utero-ovarian component. In contrast, in rabbits and mares there is no local unilateral luteolytic pathway, although a systemic pathway is present, and the uterine and ovarian vasculature are much more independent. If this concept has general application, some tentative conclusions about luteal control in the camel may be derived from studying the vascular anatomy of the utero-ovarian region in this species.

\section{Materials and Methods}

The utero-ovarian vasculature was investigated in 6 adult camels (Camelus dromedarius) using a modification of the technique used by Del Campo, Steffenhagen \& Ginther (1974). The animals 
were placed under deep general anaesthesia and heparin (50 i.u./ $\mathrm{kg}$ body weight) was infused intravenously; after $10 \mathrm{~min}$ each animal was exsanguinated via a carotid artery. The abdominal cavity was then rapidly opened in the mid-line and the aorta and vena cava were cannulated with polyethylene cannulae (15 mm o.d.). The vasculature of the entire hind end of the animal was flushed with about 20 litres of saline $(9 \mathrm{~g} \mathrm{NaCl} / 1)$ containing $0.1 \%$ sodium nitrite and 500 i.u. heparin/l, taking special care to ensure that the vessels of the genital tract were thoroughly washed. Then the whole genital tract and the broad ligaments were removed and trimmed of excess fat and connective tissue, taking care to avoid damage to the blood vessels.

In 3 camels the utero-ovarian vein and ovarian artery were cannulated with polyethylene cannulae (o.d. 1.9 and $1.22 \mathrm{~mm}$ ) and the vasculature was flushed with saline via the ovarian artery. This removed excess blood and helped to identify leaks in the vascular bed that could be closed by ligation. In other camels vasculature flushings were done via the abdominal aorta.

In 2 camels red latex (Neopren 842-A; Dupont De Nemours International, S.A., Geneva, 21 , Switzerland) was injected into the arterial system of the genitalia via the abdominal aorta, while in a $3 \mathrm{rd}$ animal warm $\left(40^{\circ} \mathrm{C}\right)$ red gelatin with red carmine $(2 \%)$ was employed. In the remaining 3 camels, 2 colours of latex were injected; red latex into the ovarian artery to fill the arterial vessels and blue latex into the uterine branch of the utero-ovarian vein to demonstrate the veins. The small leakages that occasionally occurred were controlled either with artery forceps or by ligation with surgical silk. In all camels the venous system of the utero-ovarian component was injected with blue latex via the uterine branch of the utero-ovarian vein.

After latex injection each specimen was pinned out flat in a waxed dish and left submerged in buffered formol saline for $48 \mathrm{~h}$. Then the specimen was washed in $50 \%$ methanol, and dehydrated and bleached by immersion in two changes of each of 50, 70 and $80 \%$ methanol and $87 \%, 95 \%$ and absolute ethanol. The changes were carried out at $24-\mathrm{h}$ intervals and, apart from methanol solutions which contained $10 \%$ hydrogen peroxide ( 20 volumes), each alcohol solution contained a few drops of hydrogen peroxide. The specimen was then cleared by immersion for $24 \mathrm{~h}$ in absolute ethanol/benzene $(1: 1 \mathrm{v} / \mathrm{v})$ followed by $48 \mathrm{~h}$ in benzyl benzoate. For the purpose of photography and storage the specimen was transferred into fresh benzyl benzoate.

\section{Results}

In the adult camel the ovary has the shape of a broad bean, $2 \mathrm{~cm}$ in length and $1.5 \mathrm{~cm}$ wide. The uterus was bicornuate but did not resemble that of other domestic ruminants because of its short uterine horns and lack of typical cotyledons. (There were no typical cotyledons even in one pregnant uterus.) In the specimen in which red gelatin was injected, irregular patches appeared on the endometrium numbering as many as 34 in the uterine body and 52 in the uterine horns.

\section{Arterial system (Text-fig. 1a)}

On each side the uterus was supplied by two main arteries; the first of which was the caudal uterine artery originating from the urogenital (vaginal) artery, which itself was originated from the internal iliac artery. The caudal uterine artery supplied the cervix, uterine body and caudal portion of the uterine horns. In addition the urogenital artery sends branches to the vagina, urethra and urinary bladder as vaginal, ureteric and vesicular arteries. In the camel there is no sign of a middle uterine artery which normally originates from the umbilical artery in other ruminants. The second uterine artery was the cranial uterine artery (uterine branch of ovarian artery) which supplied the more cranial parts of the uterine horns.

The ovarian artery of each side originated directly from the aorta at the level of the 5 th lumbar vertebra, approximately $3-5 \mathrm{~cm}$ before the origin of the external iliac artery. This $2 \mathrm{~cm}$ 
difference arose because in 5 of the 6 camels the left ovarian artery originated approximately 2 $\mathrm{cm}$ more caudal than the artery of the right side. The ovarian artery from its origin followed the course of the utero-ovarian vein and was highly coiled and closely applied to the vein throughout its length. This artery was divided into three branches, ovarian, uterine and oviduct, at 4-6 cm from the ovary. The ovarian artery then became tortuous and was closely applied to the cranial uterine vein (uterine branch of the utero-ovarian vein). The ovarian artery did not follow the course of the utero-ovarian vein throughout its length. In one camel the ovarian branch of the ovarian artery (main ovarian artery) was subdivided into two small branches both of which followed a convoluted course and entered the ovary separately. As this artery approached the ovary however, the degree of convolution gradually decreased and at its terminal part it was relatively straight. On either side of the uterus there were several anastomoses between the caudal and cranial uterine arteries and there was a network of anastomoses between the uterine arteries of the right and left sides, but there was no sign of arterio-venous anastomoses.

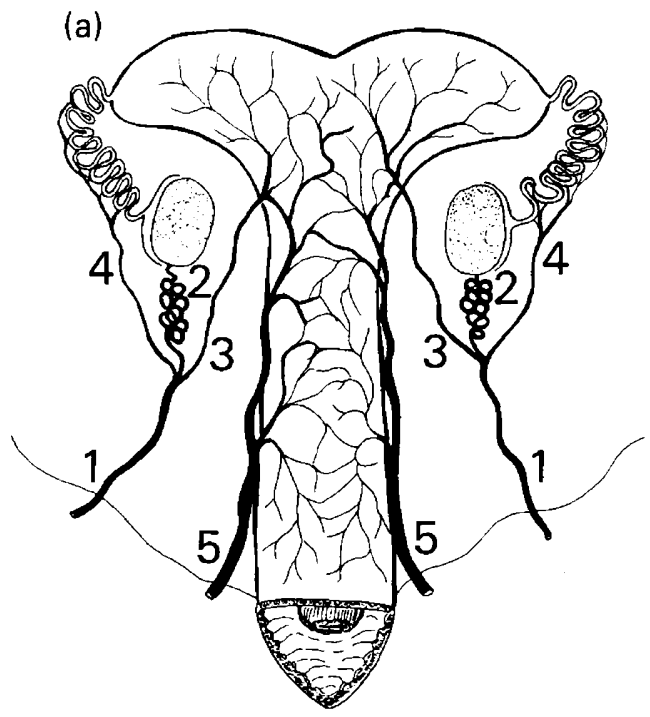

(a)

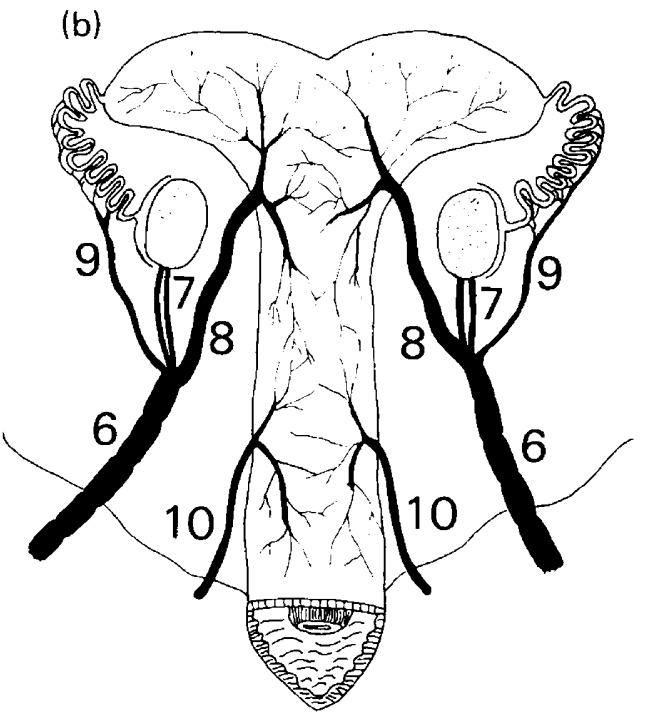

(b)

Text-fig. 1. Diagrammatic representation of the (a) arterial and (b) venous blood vessels of the uterus and ovary of the camel. 1, Ovarian artery; 2, ovarian branch of the ovarian artery; 3, uterine branch of the ovarian artery (cranial uterine artery); 4, oviduct branch of the ovarian artery; 5 , uterine artery (caudal uterine artery); 6, utero-ovarian vein; 7, ovarian branch of the utero-ovarian vein; 8, uterine branch of the utero-ovarian vein (cranial uterine vein); 9, oviduct branch of the utero-ovarian vein; 10, uterine vein (caudal uterine vein).

\section{Venous system (Text-fig. 1b)}

The main vein was the utero-ovarian vein which drained most of the uterus and all of the ovary and uterine tube. The ovarian branch of this vein was formed from 2-3 tributaries and the uterine radicle of it had at least two prominent tributaries, one from the cranial and the other from the middle part of the uterus. The greater part of the uterine blood was therefore received by the cranial uterine vein (uterine branch of the utero-ovarian vein) and venous blood of the cervix and caudal part of the uterine body was drained by the caudal uterine vein (uterine branch of the urogenital vein). The caudal uterine vein followed the course of the urogenital artery through the broad ligament and emptied into either the utero-ovarian vein just before its termination or directly into the vena cava. In one camel the utero-ovarian vein terminated in two 
branches, one entering the external iliac vein and the more prominent branch entering the vena cava. On either side one big lymphatic node (3-5 cm diameter) was always present at the site of the utero-ovarian vein termination. Irregular anastomoses were present within the broad ligament between all roots of the utero-ovarian vein (uterine, ovarian and uterine tubal veins) and extensive anastomoses were also apparent between the left and right sides. Blood from the oviduct was drained by several tiny roots which later formed 2-3 branches within the mesosalpinx and entered the utero-ovarian vein. There were valves throughout the length of the utero-ovarian vein and the distance between them varied between specimens.

\section{Discussion}

The present work indicates that, in the camel, the ovarian artery immediately after its origin, comes into close apposition with the utero-ovarian vein and follows a convoluted course over the surface of this vein. The veno-arterial contact is greatly increased because the highly tortuous ovarian branch of the ovarian artery (main ovarian artery) lies over the surface of the uterine branch of the utero-ovarian vein (cranial uterine vein). Thus the gross anatomical relationships that could allow a local unilateral transfer of the luteolytic substance(s) between the uterine venous blood and the ovarian arterial blood are present. Therefore, on anatomical grounds, it is suggested that the luteolytic pathway in the camel is of a local unilateral nature.

I thank Dr R. V. Pandit for the drawings, and Mr M. R. Shafii, Mr M. R. Masdjedi and Mr A. Mohammadi for technical assistance. This work was supported by the Shiraz University Research Council.

\section{References}

Del Campo, C.H. \& Ginther, O.J. (1973) Vascular anatomy of the uterus and ovaries and the unilateral luteolytic effect of the uterus: horse, sheep and swine. Am.J. vet. Res. 34, 305-316.

Del Campo, C.H. \& Ginther, O.J. (1974) Vascular anatomy of the uterus and ovaries and unilateral luteolytic effect of the uterus: histologic structure of the utero-ovarian vein and ovarian artery in sheep. Am. J. vet. Res. 35, 397-399.

Del Campo, C.H., Steffenhagen, W.P. \& Ginther, O.J. (1974) Clearing technique for preparation and photography of anatomic specimens of blood vessels of female genitalia. Am. J. vet. Res. 35, 303-310.

Ghazi, S.R. (1977) Feto-maternal and utero-ovarian interactions in small ruminants. Ph.D. thesis, University of Bristol.
Ginther, O.J. (1974) Internal regulation of physiological processes through local venoarterial pathways; a review. J. Anim. Sci. 39, 550-564.

Goding, J.R. (1974) The demonstration that PGF $2 \alpha$ is the uterine luteolysin in the ewe. J. Reprod. Fert. 38 , 261-271.

Inskeep, E.K. \& Butcher, R.L. (1966) Local component of utero-ovarian relationships in the ewe. J. Anim. Sci. 25, 1164-1168.

Land, R.B., Baird, D.T. \& Scaramuzzi, R.J. (1976) Dynamic studies of prostaglandin $F-2 \alpha$ in the utero-ovarian circulation of the sheep. J. Reprod. Fert. 47, 209-214.

Moor, R.M. \& Rowson, L.E.A. (1966) Local uterine mechanisms affecting luteal function in sheep. $J$. Reprod. Fert. 11, 307-310. 